\title{
Evaluation of the quality and the content of YouTube videos in Turkish on protection from coronavirus
}

\author{
(D) Mustafa Alparslan Babayiğit \\ Public Health Specialist, Private Occupational Physician, Ankara, Turkey
}

Cite this article as: Babayiğit MA. Evaluation of the quality and the content of YouTube videos in Turkish on protection from coronavirus. J Health Sci Med 2022; 5(1): 301-305.

\begin{abstract}
Aim: This study aimed to evaluate the videos in Turkish on protection from coronavirus published on YouTube in terms of general quality and content.

Material and Method: Search on YouTube website "coronavirus; protection "in line with the keyword" all the time, Turkey and the Turkish language "was held. Among the first 100 results, 63 non-repetitive and completely ad-free videos with a duration of 1-40 minutes were included in the study. The person or institution who provided the information for each video, the video length, the number of views, the number of likes and dislikes were recorded. The Global Quality Scale (GQS) was used to determine the overall quality of the videos. In addition, a scoring system was created and examined whether the information regarding the 14 rules determined by the Turkish Ministry of Health to protect the public from coronavirus was found or not in the published videos.

Results: While $50.8 \%(n=32)$ of the videos analyzed in this study were personal sharing videos for educational and informative purposes, $31.8 \%(n=20)$ of them were TV health /news programs. While $71.2 \%$ of those who provided information in the videos were medical doctors, $26.9 \%$ were out of health. When the general quality of the videos is evaluated according to the GQS scale; It was determined that $39.7 \%(n=25)$ of them were of medium quality, and $30.2 \%(n=19)$ of them were of good quality.

Conclusion: As a result of our study, it was determined that the videos that contain at least five rules and have practical application content are of better quality. In pandemic periods when preventive measures are superior to treatment, effective use of social media platforms should be ensured to raise society's awareness.
\end{abstract}

Keywords: Coronavirus, prevention, YouTube

\section{INTRODUCTION}

COVID-19, caused by the SARS-COV-2 virus, was declared a pandemic in March 2019, starting from the Wuhan province of China and spreading worldwide. Because COVID-19 is a new clinical entity, clinical information was limited in the early stages of the pandemic. However, as the process progressed, information about the disease increased (1).

As the transmission routes, clinical course, and prevention methods of the SARS-COV-2 virus were determined, the relevant experts informed the society through newspapers and television. Thus, the epidemic was tried to be brought under control. Apart from this, information is shared via the internet. Especially the free and easily accessible video-sharing site YouTube has become a preferred platform as a source of information about COVID-19 (2).
Raising public awareness about transmission routes and prevention methods is vital in essential public health (3). However, sharing on the internet is made by different people and institutions apart from the relevant experts. Therefore, apart from correct information, false and misleading messages can be given.

In order to control the epidemic and protect public health, it is necessary to monitor the content of the messages given to the society. In this study, it is aimed to evaluate YouTube videos, which are used as a source of information in protection from COVID-19, in terms of content and quality (4).

In order to control the epidemic and protect public health, it is necessary to monitor the content of the messages given to the society. In this study, it is aimed 
to evaluate YouTube videos, which are used as a source of information in protection from COVID-19, in terms of content and quality.

\section{MATERIAL AND METHOD}

This study was conducted as a YouTube research, there is no need for ethics committee approval.

"Coronavirus; In line with the keywords "protection", a search was made on 10.07.2021 for "all times, in Turkey and Turkish language". Among the top 100 results, videos with a duration of 1-40 minutes, non-repetitive and completely ad-free were included in the study for review. The researcher evaluated a total of 63 videos, which were found to meet the criteria for inclusion in the research. For each video, the person or institution providing the information, the length of the video, the number of views, the number of likes and dislikes were recorded.

The Global Quality Scale (GQS) was used to determine the overall quality of the videos. The GQS is a fivepoint Likert scale based on the quality of information, the flow of information found online, and ease of use, with 1 point very bad, 2 points bad, 3 points fair, 4 points good and 5 points excellent quality. Besides, it was examined whether the information about the fourteen rules determined within the scope of the video titled "14 Rules that will protect you and Turkey from the risk of coronavirus", prepared by the Republic of Turkey Ministry of Health to protect the public from coronavirus, is available in the published videos (5). A "MH scoring system" was created by giving 1 point if each question is in the video and 0 points if not. Accordingly, the videos were scored 0-14 points.

\section{Statistical Analysis}

Continuous variables were expressed as mean \pm standard deviation, categorical data as numbers and percentages. In the intergroup analysis of continuous variables, normality analyzes were performed with the Kolmogorov-Smirnov Goodness of Fit Test. In addition, the T-Test was used to analyze two groups when the data conformed to the normal distribution, and the Mann-Whitney U-Test was used when they did not. Analyzes were performed with IBM SPSS (Statistics Package Program for Social Sciences) version 24.0 (IBM Corporation, Armonk, NY, USA). Statistical significance level was considered as $\mathrm{p}<0.05$.

\section{RESULTS}

While $50.8 \%(n=32)$ of the YouTube videos on protection from coronavirus were personal sharing videos for educational and informational purposes,
$15.9 \%(\mathrm{n}=10)$ were TV health programs, $15.9 \%(\mathrm{n}=10)$ TV news program and $17.5 \%(n=11)$ were hospital videos. While $71.2 \%$ of the informants in the videos were medical doctors, $26.9 \%$ were non-health people. Video length median 4.0 (1-38.4) min. While the number of views was $2739(25-650,994)$, the median value on the day of publication was 234 (31-277), the number of likes was 40 (0-14000), the number of dislikes was 1 (0-570).

In $54.0 \%$ of the videos, the definition and/or complaints of the coronavirus-related flu infection were stated, $57.1 \%$ of the videos were about the route of transmission, $31.7 \%$ of the risk group was mentioned, $52.4 \%$ of them were alcohol-based in cases where handwashing is not possible. It was determined that the use of hand disinfectant or cologne was recommended. Washing hands, wearing masks, etc., in only 12 videos (19\%). It was determined that the practical applications of the precautions were included.

When the general quality status of the videos was evaluated according to the GQS scale; It was found that $39.7 \%(n=25)$ was of medium quality, 30.2\% ( $n=19)$ was of good quality, contained important information sufficiently, only some information was not included, and was beneficial for patients, whereas $20 \%$ It was determined that 0.6 of them $(n=13)$ were generally of poor quality and some of the information for patients was accurate and provided very limited benefit.

In the videos examined within the scope of the research, the most frequent hand washing (85.7\%), keeping a distance $(60.3 \%)$, avoiding close contacts $(55.6 \%)$, not touching the eyes, mouth, and nose with the hands $(49.2 \%)$ and the common cold. In addition, it was determined that the subjects wearing a mask in case of symptoms (49.2\%) were mentioned. Not sharing personal belongings $(7.9 \%)$, canceling or postponing international travels $(9.5 \%)$, washing clothes at 60 to 90 degrees (9.5\%), and frequently ventilating indoor environments (19\%) level (Table 1).

When the videos that contain at least 5 of the 14 rules determined by the Ministry of Health to protect the public from coronavirus, and the videos that express more rules in their explanations; The median values of the General quality (GQS) scale [4 (2-5)] were found to be statistically significantly higher in videos with an $\mathrm{MH}$. score of $>5$ than those with an $\mathrm{MH}$. score of $\leq 5$ $[2.50(1-3)](\mathrm{p}<0.05)$. ). It was also found that videos with an $\mathrm{MH}$ score of $>5$ included a higher rate of practical applications $(29.0 \%$ vs. $9.4 \%)(\mathrm{p}<0.05)$ (Table 2). 


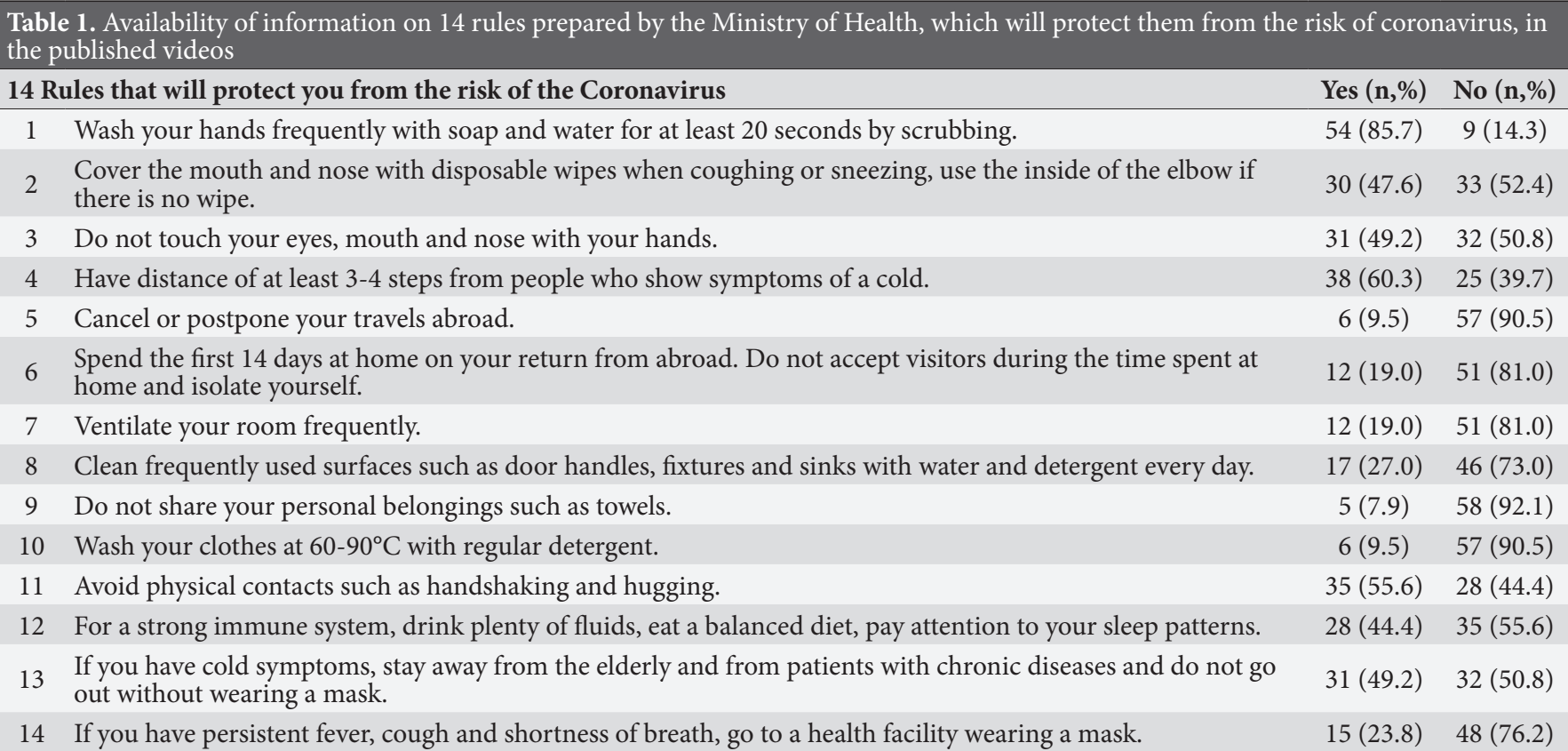

\section{Table 2. Comparison of videos on protection from coronavirus published on YouTube according to the MH scoring system}

\begin{tabular}{|c|c|c|c|}
\hline & MH score $\leq 5 \quad(n=32)$ & MH score $>5(n=31)$ & $\mathbf{p}$ \\
\hline Video length [median(min-max)] & $4.09(1-38.4)$ & $3.44(1-25.4)$ & $0.290^{*}$ \\
\hline How many days has it been on the air?[median (min-max)] & $234.5(31-273)$ & $234(194-277)$ & $0.659^{*}$ \\
\hline Number of views [median (min-max)] & $2262(25-650.994)$ & $5839(48-648.451)$ & $0.680^{*}$ \\
\hline Number of likes [median (min-max)] & $31(0-9200)$ & 76(1-14000) & $0.346^{*}$ \\
\hline Number of unlikes [median (min-max)] & $2(0-570)$ & $1(0-320)$ & $0.529^{*}$ \\
\hline General quality (GQS) scale mean score $($ Mean \pm Sd) & $2.50(1-3)$ & $4(2-5)$ & $<0.001^{\star}$ \\
\hline Category (n,\%) & & & $0.187^{\star *}$ \\
\hline TV health program & $8(25.0)$ & $2(6.5)$ & \\
\hline TV News program & $5(15.6)$ & $5(16.1)$ & \\
\hline Personal sharing & $13(40.6)$ & $19(61.3)$ & \\
\hline Hospital & $6(18.8)$ & $6(18.8)$ & \\
\hline Person giving information $(\mathrm{n}, \%)$ & & & $0.923^{\star *}$ \\
\hline Doctor & $18(56.3)$ & $2(6.5)$ & \\
\hline Other health personnel & $3(9.4)$ & $5(16.1)$ & \\
\hline Out of health & $8(25.0)$ & $19(61.3)$ & \\
\hline Newsreader & $3(9.4)$ & $3(9.7)$ & \\
\hline Practical application $(\mathrm{n}, \%)$ & & & $0.047^{\star *}$ \\
\hline No & $29(90.6)$ & $22(71.0)$ & \\
\hline Yes & $3(9.4)$ & $9(29.0)$ & \\
\hline
\end{tabular}

\section{DISCUSSION}

YouTube is a platform where millions of videos are watched every day in over 100 countries. It is used not only for entertainment purposes but also for sharing and receiving information in education, culture, and science (6). The number of shares related to health is also substantial.

In studies in the United States and Poland, it has been observed that there has been a significant increase in accessing health-related information over the internet in the last year; this rate increases even more as the level of education increases, reaching up to $98 \%$ among university graduates (7-9). Therefore, the main risk in sharing medical issues on the YouTube video-sharing site is that the person sharing can upload videos regardless of their education, medical equipment, professionalism, and purpose. For this reason, there are concerns about the accuracy, reliability, and usefulness of the shared information for society.

Videos shared on YouTube on many medical topics such as myocardial infarction, cancer, intubation, vaccination, and epidemics have been examined in terms of content 
and quality (10-16). While these studies show that video sharing on medical topics has increased in recent years, they also reflect concerns about reliability. Significantly during the pandemic process, the importance of sharing health information has increased. Since COVID-19 pneumonia is new in the literature, it has caused society and the health community concerns. Since the initial study results took time, case reports, individual experiences, and recommendations spread rapidly in magazines, newspapers, and video sharing sites. As a result of our study, it was seen that half of the Turkish internet videos providing information about COVID-19 on YouTube were individual personal sharing, the majority of them were made by doctors and were useful. Similar results have been found in previous studies on YouTube video sharing in H1N1 influenza and Ebolavirus outbreaks $(10,11)$.

In the study of D'Souza et al. (2), 113 videos containing information about COVID-19 were examined and it was seen that $69.9 \%$ of them contained useful information, and news programs had a high share of useful information.

In addition to correct, conscious informative sharing, false and misleading information about COVID-19, which is spread unconsciously or intentionally, is spreading rapidly and widely on the internet, threatening public health. In a study examining a total of 240 COVID-19 related videos in six different languages, $52.5 \%$ of the videos were found to be informative. However, it has been determined that ten percent of the videos contain medically misleading information, and independent individuals who make individual posts are responsible for $75 \%$ of the misleading content (17). Researchers have stated that health authorities such as the CDC, WHO should be included on video sharing sites.

Li et al. (4) reported that 69 COVID-19 related videos that met the criteria were included in their study, and $27.5 \%$ of them contained untrue information. They concluded that to contain the COVID-19 outbreak, public health agencies should use YouTube more effectively to provide timely and accurate information and minimize the spread of misinformation.

In the study of Ataç et al. (18), 101 Turkish and 67 English videos were examined and compared. It was found that the total content index was higher in Turkish videos. It has been observed that 23 of these videos are misleading, $65.2 \%$ of them are broadcast on news channels, and the average number of views, viewing rates, and video power scores of misleading videos are higher than valuable videos. It is worrying that misleading videos are being presented on news channels, which are watched more.

In order to prevent the spread of the epidemic, society should be informed about the identification, treatment, and isolation of the diseased people, as well as the precautions to prevent the transmission of the SARS$\mathrm{COV}-2$ virus, hygiene rules, and what to do in suspicious clinical situations. Given its popularity and easy accessibility, YouTube appears to be a virtual platform suitable for this purpose.

In the videos we examined, it was seen that basic hygiene rules and essential protective practices such as hand washing, maintaining social distance, avoiding close contacts, not touching the eyes, mouth, and nose, and wearing a mask in case of cold symptoms were mentioned. Furthermore, It was determined that the GQS scale mean scores of the videos that scored above 5 points in the MH scoring system, which we created according to the fourteen rules prepared and published by the Ministry of Health, were also significantly higher (5). In addition, it was found that practical applications in these videos were statistically significantly higher. Therefore, we believe that these videos have an essential contribution to raising public awareness.

The number of videos, changes in their content over time, and the evaluation of videos belonging to a single site as a video sharing site can be seen as limitations of our study. However, since it is an easily accessible and frequently used video-sharing site, we think it is appropriate for research and evaluation.

\section{CONCLUSION}

Video sharing sites such as YouTube attract more attention due to their visuality and are frequently used by internet users. While sharing information from these platforms can be beneficial, it also includes risks in terms of accuracy and reliability. The presence of more traditional medical institutions on these platforms and the supervision of the published videos by a scientific committee in terms of content and quality can play an essential role in being successful and effective in the fight against epidemic diseases.

\section{ETHICAL DECLARATIONS}

Ethics Committee Approval: This study was conducted as a YouTube research, there is no need for ethics committee approval.

Referee Evaluation Process: Externally peer-reviewed.

Conflict of Interest Statement: The authors have no conflicts of interest to declare.

Financial Disclosure: The authors declared that this study has received no financial support.

Author Contributions: All of the authors declare that they have all participated in the design, execution, and analysis of the paper, and that they have approved the final version. 


\section{REFERENCES}

1. Khan M, Adil SF, Alkhathlan HZ, et al. COVID-19: A global challenge with old history, epidemiology and progress so far. Molecules 2020; 26: 39.

2. D'Souza RS, D'Souza S, Strand N, Anderson A, Vogt MNP, Olatoye O. YouTube as a source of medical information on the novel coronavirus 2019 disease (COVID-19) pandemic. Glob Public Health 2020; 15: 935-42.

3. Uğraş Dikmen A, Kına HM, Özkan S, İlhan MN. Epidemiology of COVID-19: what we learn from pandemic. J Biotechinol \& Strategic Health Res 2020; 4: 29-36.

4. Li HO, Bailey A, Huynh D, Chan J. YouTube as a source of information on COVID-19: a pandemic of misinformation? BMJ Global Health 2020; 5: e002604.

5. T.C. Sağlık Bakanlığı. COVID-19 Bilgilendirme Platformu. Available online: https://covid19.saglik.gov.tr/TR-66177/ koronavirus-riskine-karsi-14-kural-kamu-spotu.html (Accessed date:05.08.2021)

6. Wikipedia. YouTube. Available online: https://tr.wikipedia.org/ wiki/YouTube. (Accessed date:05.08.2021).

7. Kwan G, Shaw JA, Murnane L. Internet usagewithin healthcare: how college students use the internet to obtain health information. J Consumer Health Internet Vol 2019; 23: 366-77.

8. National Cancer Institute. 2017. "Health Information National Trends Survey, HINTS-FDA Cycle2.” Last updated 2017; Accessed date:26.10.2020. https://hints.cancer.gov/viewquestions-topics/ question-details.aspx?PK_Cycle=9\&qid=757.

9. Paré G, Malek JN, Sicotte C, Lemire M. Internet as a source of health information and its perceived influence on personal empowerment. IJHISI 2009; 4: 1-18.

10. Pathak R, Poudel DR, Karmacharya P, et al. YouTube as a source of information on Ebola virus disease. N Am J Med Sci 2015; 7: 306-9.

11.Pandey A, Patni N, Singh M, Sood A, Singh G. YouTube as a source of information on the H1N1 influenza pandemic. Am J Prev Med 2010; 38: e1-3.

12.Pant S, Deshmukh A, Murugiah K, Kumar G, Sachdeva R, Mehta JL. Assessing the credibility of the "YouTube approach" to health information on acute myocardial infarction. Clin Cardiol 2012; 35: 281-5.

13. Hassona Y, Taimeh D, Marahleh A, Scully C. YouTube as a source of information on mouth (oral) cancer. Oral Dis 2016; 22: 202-8.

14. Syed-Abdul S, Fernandez-Luque L, Jian WS, et al. Misleading health-related information promoted through video-based social media: anorexia on YouTube. J Med Internet Res 2013; 15: e30.

15.Donzelli G, Palomba G, Federigi I, et al. Misinformation on vaccination: A quantitative analysis of YouTube videos. Hum Vaccin Immunother 2018; 14: 1654-9.

16. Babayiğit M, Babayiğit MA. Evaluation of YouTube videos related to intubation in the pandemic process in terms of quality and content in Turkish and English. Anatolian Curr Med J 2021; 3; 234-8.

17. Dutta A, Beriwal N, Van Breugel LM, et al. YouTube as a source of medical and epidemiological information during COVID-19 pandemic: a cross-sectional study of content across six languages around the globe. Cureus 2020; 12: e8622.

18. Ataç Ö, Özalp YC, Kurnaz R, Güler OM, İnamlık M, Hayran O. Youtube as an information source during the coronavirus disease (COVID-19) pandemic: evaluation of the Turkish and English content. Cureus 2020; 12: e10795. 